\title{
A territorialização como instrumento para formação em saúde a partir de uma experiência de extensão popular
}

Jeane Constantino Pereira ${ }^{1}$, Erika Valeska da Costa Alves $^{2}$, Artemis Rodrigues Dias ${ }^{3}$, Dailton Alencar Lucas de Lacerda ${ }^{4}$

\begin{abstract}
Resumo
Este texto relata a importância da territorialização como instrumento para a formação em saúde a partir de uma experiência de extensão popular vinculada à Universidade Federal da Paraíba. Trata-se de um relato de experiência, com abordagem qualitativa, de atividade de territorialização, baseado na sistematização de experiências segundo Oscar Jara. A atividade foi vivenciada por estudantes, professores e trabalhadores da atenção básica, como parte do Programa Educação Popular em Saúde do Trabalhador (PEPST), o qual tem como eixo teórico-metodológico a Educação Popular em Saúde. O trabalho teve como cenário de prática a comunidade do Grotão, localizada no município de João Pessoa-PB. Os estudantes foram divididos em grupos e acompanhados por profissionais da equipe de saúde. As impressões foram relatadas em um diário de campo. Também foram realizadas entrevistas com ex-participantes do PEPST. A partir dessa experiência ficou evidente que a territorialização contribui para a reorientação da formação em saúde e para a integração ensinoserviço-comunidade, apresentando-se também como um subsídio para o planejamento de ações estratégicas capaz de considerar as iniquidades sociais inerentes ao território.
\end{abstract}

\section{Palavras-chave}

Território sociocultural. Atenção Primária à Saúde. Educação em saúde.

\footnotetext{
1 Mestra em Neurociência Cognitiva e Comportamento pela Universidade Federal da Paraíba, Brasil; pesquisadora integrante do Grupo de Estudos, Pesquisa e Extensão Saúde e Comunidade (GEPEX-SC) da Universidade Federal da Paraíba, Brasil. E-mail: jehconstantino@gmail.com.

2 Doutoranda em Gerontologia na Universidade Estadual de Campinas, São Paulo, Brasil; pesquisadora integrante do Grupo de Estudos, Pesquisa e Extensão Saúde e Comunidade (GEPEX-SC) da Universidade Federal da Paraíba, Brasil. E- mail: erika.valeska@yahoo.com.br.

${ }^{3}$ Graduada em Fisioterapia pelo Centro Universitário de João Pessoa, Paraíba, Brasil; coordenadora adjunta do Programa Mais Saude da Universidade Federal da Paraíba, Brasil. E-mail: artemis.fisio@ hotmail.com.

${ }^{4}$ Doutor em Ciências da Saúde pela Universidade Federal do Rio Grande do Norte, Brasil; professor adjunto no Centro de Ciências da Saúde da Universidade Federal da Paraíba, Brasil; coordenador de Educação Popular da Pró-Reitoria de Extensão e Assuntos Comunitários na mesma instituição. E-mail: dailtonlacerda@gmail.com.
} 


\title{
The territorialization as a tool for healthcare education based on an experience of popular extension
}

Jeane Constantino Pereira ${ }^{5}$, Erika Valeska da Costa Alves ${ }^{6}$, Artemis Rodrigues Dias ${ }^{7}$, Dailton Alencar Lucas de Lacerda ${ }^{8}$

\begin{abstract}
This text reports the importance of territorialization as a tool for healthcare education based on an experience of popular extension conducted by Federal University of Paraíba. A qualitative study on territorialization was designed based on systematic experiences by Oscar Jara. The theoretical and methodological approaches of Popular Health Education was used in Popular Education in Occupational Health Program (PEPST) where undergraduate students, professors and primary health care workers were subjected to territorialization activity in Grotão community located in João Pessoa, State of Paraíba, Brazil. The undergraduate students were divided into groups and activity of each groups were performed under the guidance of workers Primary Health Care. The reflective experiences gained were daily recorded in field. Simultaneously interviews were also conducted from former PEPST participants. The observations provided from the territorialization activity inferred that territorialization has a major impact on re-orientation of health care education and contributes to integration of education, health care service and community. Preplanning strategy measures framed using territorialization provided better frameworks of social inequalities in a local community.
\end{abstract}

\section{Keywords}

Sociocultural territory. Primary Health Care. Health education.

\footnotetext{
5 Master in Cognitive Neuroscience and Behavior, Federal University of Paraíba, Brazil; researcher member of the Group of Studies, Research and Extension Health and Community (GEPEX-SC), Federal University of Paraíba, Brazil. E-mail: jehconstantino@gmail.com.

${ }^{6} \mathrm{PhD}$ student in Gerontology, State University of Campinas, São Paulo, Brazil; researcher member of the Group of Studies, Research and Extension Health and Community (GEPEX-SC), Federal University of Paraíba, Brazil. E- mail: erika.valeska@yahoo.com.br.

${ }^{7}$ Graduated in Physiotherapy, University Center of João Pessoa, Paraíba, Brazil; assistant coordinator of the More Health Program, Federal University of Paraíba, Brazil. E-mail: artemis.fisio @ hotmail.com.

${ }^{8} \mathrm{PhD}$ in Health Sciences, Federal University of Rio Grande do Norte, Brazil; adjunct professor at the Health Sciences Center at the Federal University of Paraíba, Brazil; Popular Education coordinator of the Dean of Extension and Community Affairs at the same institution. E-mail: dailtonlacerda@gmail.com.
} 


\section{Introdução}

O território pode ser compreendido como um espaço geográfico único, organizado de forma descentralizada no âmbito do Sistema Único de Saúde (SUS) e que executa ações estratégicas de vigilância, prevenção, proteção, promoção e recuperação da saúde (BRASIL, 2017).

Ao se olhar para o território na perspectiva de produção da saúde, deve-se considerálo um espaço vivo, capaz de produzir saúde e que precisa passar por um diagnóstico epidemiológico que identifique fatores e condições pertinentes ao processo de saúde e doença de uma determinada região (SUCUPIRA, 2003). Dessa forma, a territorialização também pode contribuir com a gestão e o planejamento de intervenções a partir da realidade local (FARIA, 2013).

A territorialização compreende um processo em que um espaço geográfico é analisado em todas as suas dimensões, a fim de que se obtenha uma visão mais ampla do seu contexto e das atividades humanas nele desenvolvidas (MONKEN; BARCELLOS, 2005). Com isso, a territorialização vai além da análise dos limites geográficos e busca entender o território e, ainda, as necessidades de quem nele habita. Assim, os resultados desse processo dialogam com as diretrizes da Atenção Primária à Saúde (APS) (CAMARGOS; OLIVER, 2019).

A Educação Popular em Saúde (EPS) pode ser compreendida como um modo particular de identificar e superar os problemas de saúde a partir do diálogo, respeito e valorização dos saberes populares, considerando como escopo teórico os fundamentos de Paulo Freire (VASCONCELOS, 2007).

Nesse contexto, no ano de 2013, foi publicada a Política Nacional de Educação Popular (PNEPS-SUS) com o intuito de fortalecer o SUS por estimular, dentre outros aspectos, a participação popular, o cuidado, a formação e as práticas educativas em saúde (BRASIL, 2013).

A partir do entendimento de que um dos papéis da Universidade é unir o ensino e a pesquisa à sociedade, a extensão de caráter popular propõe um trabalho social útil e pautado pela troca de saberes, diálogo, respeito às diferenças, solidariedade entre as pessoas e responsabilidade social (MELO NETO, 2014).

Assim, para além do que é visto em sala de aula - e aproximando os estudantes da realidade, por meio de um processo formativo crítico, reflexivo, vivencial e interdisciplinar surge o Programa Educação Popular em Saúde do Trabalhador (PEPST). O Programa é 
vinculado à Universidade Federal da Paraíba (UFPB) e constitui-se como atividade de extensão universitária que tem como fio condutor a EPS. Sua finalidade é a promoção da saúde do trabalhador numa perspectiva crítica e integrativa, articulando projetos cujas ações se desenvolvem no âmbito da Atenção Primária à Saúde (APS).

Nesse sentido, o objetivo deste trabalho é relatar a importância da territorialização como instrumento para a formação em saúde a partir de uma experiência de extensão popular vinculada à UFPB.

\section{Metodologia}

Trata-se de um relato de experiência sobre uma atividade de territorialização, com abordagem qualitativa. A atividade foi vivenciada por 30 estudantes, que cursavam do primeiro ao último ano de graduação de cursos de diversas áreas: fonoaudiologia, fisioterapia, nutrição, medicina, enfermagem, direito, serviço social, psicologia e odontologia. Além deles, participaram também professores e trabalhadores da Atenção Básica em Saúde.

O PEPST tem como eixo teórico-metodológico a EPS, e busca promover a construção de processos de reflexão, problematização e diálogo entre trabalhadores de diferentes categorias e estudantes universitários.

A atividade de territorialização precedeu as ações do PEPST, compostas por reuniões teóricas e por visitas semanais aos trabalhadores, que ocorriam no domicílio ou no ambiente de trabalho.

O processo de territorialização ocorreu com o reconhecimento da comunidade, construído em parceria com a Unidade Integrada de Saúde da Família (UISF) do Grotão, localizada na zona sul do município de João Pessoa-PB e vinculada ao distrito sanitário II. A palavra comunidade é derivada do latim e significa “o mesmo". Dentre inúmeros conceitos, considerou-se para este estudo que comunidade é um espaço de interação entre as pessoas que estão em determinada localidade e apresentam necessidades semelhantes, passíveis de serem alcançadas por meio da colaboração dos envolvidos (GRIEBELER OLIVEIRA et al., 2013).

A organização dos extensionistas na atividade de territorialização foi feita considerando a demarcação da área de abrangência da UISF Grotão. Os estudantes foram divididos em três grupos correspondentes às áreas I, II e III da referida unidade, sendo que cada grupo foi acompanhado por um profissional de saúde da UISF. 
A atividade de territorrialização foi orientada por um roteiro, contendo eixos norteadores, dentre os quais: delimitação do mapa geofísico do território; estudo sóciohistórico e cultural, através da observação e de entrevistas com sujeitos do território (moradores e lideranças comunitárias); identificação do perfil social, econômico e cultural dos seus habitantes; identificação dos equipamentos sociais existentes na área; diagnóstico socioambiental e sanitário; identificação do perfil demográfico e de habitação da população; identificação das principais atividades produtivas do território; apropriação das condições de saúde, sanitárias e epidemiológicas da comunidade; análise do papel da UISF no território.

As informações obtidas foram sistematizadas em diário de campo, como sugerido no trabalho Para Sistematizar Experiências, de Holliday (2006), com o objetivo de compreender mais profundamente as experiências realizadas, visando o aprimoramento da prática. Além disso, a sistematização também possibilita o compartilhamento dos aprendizados adquiridos e a reflexão teórica dos ensinamentos oriundos de práticas sociais concretas.

Cada diário de campo continha tópicos (objetivos, metodologia, público-alvo e resultados identificados) que eram preenchidos por uma dupla de estudantes, a fim de coletar impressões e reflexões próprias logo após a atividade. Dessa forma, a memória da experiência foi preservada, para que possa ser resgatada sempre que necessário. A fim de complementar as experiências descritas nos diários, foram realizadas também entrevistas com ex-participantes do PEPST.

\section{Resultados e Discussão}

Essa experiência de extensão utilizou a territorialização como uma ferramenta estratégica no desenvolvimento metodológico das suas ações.

Observou-se que a comunidade possuía diversos equipamentos sociais, tais como: unidades de saúde, escolas, igrejas, associações de moradores, rádio comunitária, pequenos estabelecimentos comerciais, entre outros. Um grande destaque da localidade era a feira livre, a qual oferecia grande variedade de produtos a preços acessíveis. Embora a comunidade dispusesse de energia elétrica, grande parte ainda carecia de pavimentação de vias e de saneamento básico. Outra característica era a presença do tráfico de drogas e da violência na região. Vale destacar que neste território existia um assentamento populacional de moradores sem-teto, que por configurar área de influência, recebia assistência da unidade de saúde local. Tais condições tornavam esses moradores mais vulneráveis a iniquidades 
sociais e de saúde.

A territorialização possibilita a reorientação das práticas de trabalho em saúde, sendo um instrumento estratégico de atuação no território, que possibilita a compreensão ampliada da dinâmica do território na Atenção Básica (JUSTO et al., 2017). O participante da experiência, estando diante de todo esse cenário, passa a observar cada detalhe e compreender melhor a situação de vulnerabilidade do território e da sua população.

Em relação à percepção dos estudantes extensionistas, a atividade proporcionou uma visão crítica e minuciosa sobre as condições sociais, econômicas e de saúde do local, conforme abordado a seguir na fala de uma nutricionista ex-extensionista do projeto:

[...] passei a questionar o que tinha ao redor. Qual o acesso desse moradortrabalhador à alimentação? Qual era sua rede de apoio? Qual o acesso deles à educação? Lazer? Transporte? Não era apenas saber que não tinha acesso, mas porque não o tinha. E a territorialização me trazia essas respostas. (A.M.B, 27 anos, nutricionista ex-extensionista do projeto).

Assim sendo, os sujeitos da ação passam a compreender o cotidiano daquele espaço geopolítico e social, valorizando a experiência de vida da população daquele território, podendo atuar e intervir com resolutividade nas suas ações de saúde. Além disso, conhecer o território fez com que os estudantes extensionistas se tornassem mais autônomos e seguros para circularem naquela localidade.

Por parte dos moradores da comunidade, a atividade foi uma oportunidade de conhecer os participantes e os propósitos das ações a serem desenvolvidas. Como mencionado na fala de uma das Agentes Comunitárias de Saúde (ACS) que acompanhou um dos grupos: “[...] só a gente sair na área, olhar as coisas, ver, perguntar [...] a comunidade vê, pergunta quem é elas [extensionistas do projeto], se interessa pelo trabalho [...] muitas vezes a comunidade até se alegre também, acha que é pra ajudar mais. (D.M.S, 61 anos, Agente Comunitária de Saúde)

Segundo Faria (2018), a territorialização vai além da delimitação do território, devendo existir também uma relação de vínculo, além de contribuir para o fortalecimento da articulação ensino-serviço-comunidade. Essa atividade proporciona aos estudantes extensionistas uma melhor compreensão das necessidades e potencialidades locais e serve como norte para o planejamento de um modelo de ações voltadas para realidade local.

A partir da territorialização realizada no PEPST, foi possível planejar de forma adequada as ações futuras, como reuniões teóricas e visitas aos trabalhadores, os quais pertenciam a diversas categorias de ocupação (formal ou informal). O uso da EPS, baseada 
no pensamento freiriano, é expresso por uma pedagogia pautada na valorização de experiências, no diálogo e na ação comum entre os sujeitos (STOTZ; DAVID; WONG-UN, 2005). Esses elementos nortearam desde o planejamento até a execução da territorialização com a equipe de saúde.

A valorização da experiência dos ACS acerca do território permitiu uma visita de forma segura e planejada, a partir do olhar de quem mora e trabalha na comunidade e, portanto, conhece a história e a dinâmica do lugar para além dos dados sociodemográficos. O diálogo com a comunidade foi iniciado já na territorialização e mediado pela equipe de saúde, já reconhecida pela comunidade como parceira. Este elemento foi alimentado nas ações de visitas posteriormente realizadas pelos estudantes extensionistas aos trabalhadores da comunidade.

É importante destacar que vivências baseadas na realidade do território possibilitam aos estudantes conhecerem as autênticas condições de vida e de saúde da comunidade, propiciando o entendimento da saúde como um processo multideterminado e permitindo aos estudantes irem além de sua área de formação específica (RIOS; CAPUTO, 2019).

Diante disso, entende-se que a territorialização é um importante instrumento para a reorientação da formação em saúde, assim como, para o fortalecimento da integração ensinoserviço-comunidade. Além de ser um subsídio para o planejamento de ações estratégicas que levem em consideração as iniquidades sociais inerentes àquele território.

\section{Considerações finais}

A territorialização é uma ferramenta de suma importância para o desenvolvimento das ações de extensão na comunidade norteadas pela EPS. A experiência possibilitou o reconhecimento do território de uma forma mais ampliada, favoreceu o diálogo com a comunidade e permitiu a construção de vínculos entre os extensionistas, a equipe de saúde e os moradores. Fez com que os sujeitos que dela participaram se apropriassem daquele espaço, antes desconhecido, guiando ações futuras desenvolvidas pelo PEPST.

Assim sendo, as atividades realizadas no âmbito da extensão universitária, tais como a territorialização, proporcionam um processo de aprendizado dinâmico, crítico e reflexivo que ressignifica a formação de futuros trabalhadores de saúde. 


\section{Referências}

BRASIL. Ministério da Saúde. Política Nacional de Educação Popular em Saúde no SUS. Brasília, DF: MS, 2013.

BRASIL. Ministério da Saúde. Portaria nº 2.436, de 21 de setembro de 2017. Diário Oficial da União, Brasília, DF, Seção 1, n. 183, p. 68-76, set. 2017.

CAMARGOS, M. A.; OLIVER, F. C. Uma experiência de uso do georreferenciamento e do mapeamento no processo de territorialização na Atenção Primária à Saúde. Saúde em Debate, Rio de Janeiro, v. 43, n. 123, p. 1.259-1.269, out./dez. 2019. Doi: 10.1590/0103-1104201912321.

FARIA, R. M. A territorialização da atenção primária à saúde do SUS: avaliação dos resultados práticos implementados numa cidade da região sul do Brasil. Hygeia, Uberlândia, v. 14, n. 29, p. 89-104, out. 2018. Doi: 10.14393/Hygeia142908.

FARIA, R. M. A territorialização da atenção primária à saúde no Sistema Único de Saúde e a construção de uma perspectiva de adequação dos serviços aos perfis do território. Hygeia, Uberlândia, v. 6, n. 16, p. 131-147, jun. 2013.

GRIEBELER OLIVEIRA, S. et al. Discussões sobre o conceito de comunidade relacionado à atuação do enfermeiro: relato de experiência. Avances en Enfermería, Bogotá, v. 31, n. 1, p. 133-140, jun. 2013.

HOLLIDAY, O. J. Para sistematizarexperiências. Tradução de Maria Viviana V. Resende. 2. ed. Revista. Brasília: MMA, 2006.

JUSTO, L. G. et al. A territorialização na atenção básica: um relato de experiência na formação médica. Interface, Botucatu, v. 21, supl. 1, p. 1.345-1.354, 2017. Doi: 10.1590/1807-57622016.0512.

MELO NETO, J. F. Extensão popular. João Pessoa: Editora da UFPB, 2014.

MONKEN, M.; BARCELLOS, C. Vigilância à saúde e território utilizado: possibilidades teóricas e metodológicas. Cadernos de Saúde Pública, Rio de Janeiro, v. 21, n. 3, p. 898906, maio/jun. 2005. Doi: 10.1590/S0102-311X2005000300024.

RIOS, D. R. S.; CAPUTO, M. C. Para além da formação tradicional em saúde: experiência de educação popular em saúde na formação médica. Revista Brasileira de Educação

Médica, Brasília, v. 43, n. 3, p. 184-195, jul. 2019. Doi: 10.1590/1981$52712015 \mathrm{v} 43 \mathrm{n} 3 \mathrm{rb} 20180199$.

STOTZ, E. N.; DAVID, H. M. S. L.; WONG- UN, J. A. Educação popular e saúde: trajetória, expressões e desafios de um movimento social. Revista APS, Juiz de Fora, v. 8, n.1, p. 49-60, jan./jun. 2005.

SUCUPIRA, A. C. Marco conceitual da promoção da saúde no PSF. Sanare, Sobral, v. 4, n. 1, p. 11-14, jan./mar. 2003. 
VASCONCELOS, E. M. Educação popular: instrumento de gestão participativa dos serviços de saúde. In: BRASIL. Ministério da Saúde. Caderno de Educação Popular e Saúde.

Brasília, DF: MS, 2007. p. 18-30.

Submetido em 20 de março de 2020.

Aprovado em 19 de maio de 2020. 